Metodički obzori 6(2011)2

Original scientific article

UDK: 371.13:[378.7:811.131.1]

Received: 23. 11. 2010.

\title{
PRESENTAZIONE DEL MODELLO DI FORMAZIONE PER INSEGNANTI OPERANTI IN AREE PLURILINGUISTICHE CON PRESENZA DI LINGUA MINORITARIA
}

\author{
Doc. dr. Nives Zudič Antonič, \\ Università del Litorale, \\ Facoltà di Studi Umanistici, Capodistria (Slovenia) \\ e-mail: nives.zudic.antonic@fhs.upr.si
}

\begin{abstract}
Sintesi
Il presente intervento intende illustrare i risultati della ricerca internazionale svolta nell'ambito del progetto Comenius 2.1 INFO - "Individuazione di un modello di formazione per insegnanti operanti in aree plurilinguistiche con presenza di lingua minoritaria". Nell'intervento verrà presentata la ricerca svolta nell'ambito del progetto che ha portato all' individuazione e allo sviluppo di un modello di formazione per insegnanti in servizio che consenta all'insegnante di disporre di strumenti didattici e metodologici, conoscenze, atteggiamenti e strategie tali da realizzare al meglio la pratica didattica e promuovere a pieno la possibilità di crescita degli alunni parlanti lingue minoritarie.

Il profilo professionale elaborato sulla base dello studio condotto ha considerato gli elementi di qualificazione che fanno da comune denominatore per tutti i sistemi scolastici plurilingui con presenza di lingua minoritaria e hanno permesso di individuare dei moduli di specializzazione ed approfondimento di problematiche e tematiche che sono state l'oggetto di studio del corso di formazione europeo.
\end{abstract}

Parole chiave: lingua minoritaria, italiano, Comenius 2.1, formazione dei docenti, docenti, plurilinguismo, corso di formazione.

\section{Introduzione}

Il presente intervento intende illustrare i risultati della ricerca internazionale svolta nell' ambito del progetto Comenius 2.1 INFO - Individuazione di un modello di formazione per insegnanti operanti in aree plurilinguistiche con presenza di lingua minoritaria (2005-07). La finalità del progetto era quella di individuare un modello di formazione basato su indicatori di qualità, correlato a nuove competenze professionali, rispondente alle specificità delle aree plurilinguistiche e corrispondente a forme di insegnamento e didattica plurilingue.

Nell'ambito del progetto sono stati rilevati i bisogni educativi del contesto socioculturale, quelli di sistema e quelli del personale insegnante. L'analisi dei bisogni 
ha permesso l'individuazione del quadro di riferimento delle conoscenze, abilità e atteggiamenti richiesti agli insegnanti che operano in contesti plurilinguistici.

Il profilo professionale elaborato sulla base dello studio condotto ha considerato gli elementi di qualificazione che fanno da comune denominatore per tutti i sistemi scolastici plurilingui con presenza di lingua minoritaria e hanno permesso di individuare dei moduli di specializzazione ed approfondimento di problematiche e tematiche che sono state l'oggetto di studio del corso di formazione europeo.

\section{Il progetto internazionale Comenius 2.1: \\ "INFO - Individuazione di un modello di formazione per insegnanti operanti in aree plurilinguistiche con presenza di lingua minoritaria"}

Il progetto nasce per far fronte ad un'esigenza reale e comune degli insegnanti appartenenti alle minoranze linguistiche storiche europee. Il bilinguismo, o a volte il plurilinguismo, che caratterizza i parlanti di una minoranza linguistica e influenza le competenze linguistiche dei parlanti stessi, implica delle conoscenze e degli approcci particolari da parte degli insegnanti che operano nelle istituzioni scolastiche.

Negli ultimi anni, grazie ai movimenti di autopromozione da parte delle minoranze e grazie ad una crescente sensibilità nei confronti delle lingue meno diffuse, considerate finalmente come ricchezza nel panorama culturale europeo, la tutela delle minoranze si è rafforzata. Rimangono in ogni modo molte differenze fra i sistemi normativi europei che consentono in maniera molto diversificata l'insegnamento della lingua materna all'interno delle istituzioni scolastiche.

Alcuni paesi europei prevedono l'insegnamento della lingua madre fin dalla scuola primaria, altri considerano detto insegnamento alla stregua dell'opzionalità o della progettualità extracurricolare, altri ancora ne escludono completamente la pratica.

Nell'introdurre la lingua minoritaria nei sistemi scolastici, fino ad ora a carattere monolingue con l'insegnamento di tutte le materie perlopiù nella lingua nazionale, l'insegnante ha bisogno di competenze e conoscenze particolari che gli permetta di promuovere la lingua materna in un contesto curriculare plurilingue. Se l'alfabetizzazione nella lingua madre per una minoranza non è più un obiettivo realisticamente ipotizzabile in un contesto plurilinguistico come quello attuale, i sistemi scolastici delle aree in presenza di lingua meno diffusa si trovano a dover trovare ed attuare una didattica ed una metodologia che consenta di promuovere la lingua materna in primis e nel contempo anche le altre lingue presenti nel territorio, nel contesto sociale e culturale di riferimento, così come le altre lingue europee.

Il progetto si prefigge di individuare e realizzare un modello di formazione per insegnanti in servizio, che, evidenziando gli elementi di qualità di un percorso professionalizzante, consentano all'insegnante di disporre di strumenti didattici e metodologici, conoscenze e atteggiamenti e di utilizzare approcci e strategie, tali da realizzare al meglio la pratica didattica e promuovere appieno le possibilità di crescita degli alunni parlanti lingue meno diffuse. 
Il progetto si sviluppa nella collaborazione fra diversi partner europei ${ }^{1}$ operanti in aree con presenza di minoranze linguistiche. All'inizio del progetto il gruppo di lavoro ha predisposto gli strumenti della Ricerca, attraverso i quali si è inteso rilevare i bisogni degli insegnanti e del contesto socioculturale. Sono stati somministrati questionari per dirigenti, docenti, genitori e altri portatori d'interesse, sono stati realizzati gruppi di discussione e sono stati raccolti dati relativi alle attività e ai progetti svolti in lingua minoritaria nelle istituzioni scolastiche di undici aree plurilinguistiche. Con i risultati, emersi dall'analisi dei bisogni del corpo docente, dei dirigenti e dei portatori di interesse, è stato possibile elaborare un modello di formazione. Il modello vuole proporre alle istituzioni che formano il personale insegnante in servizio delle Linee guida per strutturare un percorso di formazione specifico per insegnanti di scuola primaria operanti in aree plurilinguistiche con presenza di lingua minoritaria. La guida vuole orientare i contenuti del corso verso le reali necessità degli insegnanti che desiderano o devono promuovere e realizzare un progetto, un'attività o un percorso in lingua minoritaria, oppure per coloro che si devono confrontare con un approccio didattico plurilingue tenendo conto della madrelingua degli alunni (qualora si tratti di lingua minoritaria) o anche solo per coloro che desiderano competere professionalmente con standard di insegnamento europei orientati sempre più verso una didattica del plurilinguismo.

\section{La ricerca}

\section{Situazione, bisogni e aspettative dei docenti di lingua minoritaria che insegnano in aree plurilinguistiche}

Nell'abito del progetto europeo (Comenius 2.1) INFO è stata realizzata una ricerca con 1'intento di analizzare la situazione degli insegnanti di alcune aree linguistiche in Europa ed in particolare con l'obiettivo di individuare i bisogni degli insegnanti di lingua minoritaria nelle scuole primarie.

I risultati della ricerca sono stati gli elementi di discussione sui quali il gruppo di ricerca ha costruito un modello sperimentale di formazione in servizio.

Nell'ambito della ricerca sono state analizzate in totale undici aree plurilinguistiche: le valli ladine di Gardena, Badia e Fassa (Trentino Alto-Adige); le scuole in Friuli in cui si insegna il friulano, lo sloveno, il timavese, il sauro (aree germanofone) - (Friuli Venezia Giulia); le scuole sarde con 1'insegnamento del sardo, del catalano (Alghero) e del tabarchino (Isola di San Pietro) - (Sardegna); le scuole italiane in Slovenia e le scuole slovene con insegnamento della lingua italiana come L2

\footnotetext{
${ }^{1}$ I partner del progetto: Istituto Pedagogico Ladino, Bolzano, Italia (Istituto Coordinatore); Ministero della Pubblica Istruzione, Ufficio Affari Internazionali, Roma, Italia; Sorastanza de la scoles de Fascia, Val di Fassa, Italia; Direzione Scolastica Regionale Friuli Venezia Giulia, Trieste, Italia; Istituto Regionale di Ricerca Educativa Friuli Venezia Giulia, Trieste, Italia; Istituto Regionale di Ricerca Educativa della Sardegna, Cagliari, Italia; Università del Litorale, Facoltà di Studi Umanistici, Capodistria, Slovenia; Padagogische Akademie Klagenfurt, Austria; Denbighshire County Council, Denbigh/Galles, Regno Unito.
} 
- (Litorale Slovenia); le scuole slovene in Carinzia (Austria) e le scuole gallesi in Galles (Gran Bretagna).

La metodologia della ricerca prevedeva innanzitutto la somministrazione di questionari ai dirigenti scolastici (in totale 41questionari), ai docenti (in totale 281 questionari), ai portatori di interesse (in totale 39 questionari), quali genitori, rappresentanti politici e degli enti culturali. In un secondo tempo sono stati realizzati dei gruppi di discussione, con le stesse rappresentanze scolastiche, politiche e amministrative, con le quali si sono voluti evidenziare problemaliche, aspettative e percezioni, oltre a quelle previste nei questionari. Nel contempo è stata realizzata una mappatura delle attività e dei progetti in lingua minoritaria attuati nell'anno scolastico 2004-2005. I risultati di tale indagine permettono di disporre di numerosi dati sulle metodologie e sui contenuti dell'insegnamento della lingua minoritaria nelle scuole primarie.

Per quanto riguarda la nostra realtà alla ricerca INFO hanno partecipato 8 scuole elementari della zona costiera; le tre scuole lingua d'insegnamento italiana (SE Pier Paolo Vergerio il Vecchio di Capodistria; SE Dante Alighieri di Isola; SE Vincenzo De Castro di Pirano) e 5 scuole con lingua d'insegnamento slovena (SE (Osnovna šola) Elvira Vatovec Prade; SE (Osnovna šola) Oskar Kovačič Škofije; SE (Osnovna šola) Dušan Bordon Semedela; SE (Osnovna šola) Vojka Šmuc Isola; SE (Osnovna šola) Ciril Kosmač Piran).

Nel Litorale sono stati somministrati pertanto 8 questionari per i dirigenti scolastici, 40 questionari per gli/le insegnanti (10 docenti per ogni istituto italiano e 10 docenti di tutti i 5 istituti sloveni -2 per istituto), 5 questionari per i portatori di interesse (genitori, sindaci e dirigenti di enti culturali). I questionari insegnanti e per portatori di interesse sono stati somministrati ad un campione di 10 docenti per istituto e di 5 portatori di interesse. I risultati dei dirigenti invece rispecchiano completamente la realtà delle scuole italiane in Slovenia in quanto sono stati somministrati a tutti i dirigenti dell'area del Litorale.

Al gruppo di discussione hanno partecipato 11 persone, alcune già coinvolte nella prima fase (somministrazione dei questionari), altre coinvolte per la prima volta nell'indagine.

\section{I questionari, le domande al gruppo di discussione, le schede di mappatura}

I questionari erano composti da un minimo di 12 domande (per portatori di interesse) ad un massimo di 27 domande (per i docenti). Il questionario era suddiviso in diverse parti che rispecchiavano argomenti diversi. Una prima parte prevedeva la (auto)valutazione delle competenze linguistiche e dei risultati dell'attività didattica, una seconda parte mirava a raccogliere dati sulle metodologie di insegnamento, un'ulteriore parte era poi incentrata sulla politica linguistica ed infine una parte verteva sulle aspettative, percezioni e bisogni degli insegnanti e delle aree plurilinguistiche, la parte forse più interessante e innovativa della ricerca.

Le domande erano strutturate a risposta chiusa con una certa flessibilità nei gradi di risposta. Solo per una minima parte delle domande era prevista la risposta sì/no, 
mentre la maggioranza delle domande prevedeva di graduare la risposta da un minimo di uno ad un massimo di sette.

A conclusione del questionario era prevista la possibilità di inserire idee, suggerimenti o solamente le proprie impressioni sull'universo delle lingue minoritarie.

Il gruppo di discussione è stato invece invitato, in occasione di una riunione convocata appositamente nelle diverse aree, ad esprimersi sulle stesse tematiche, rispondendo a delle domande aperte alle quali i partecipanti potevano esprimersi liberamente.

Le attività ed i progetti realizzati in lingua minoritaria e/o con più lingue sono stati raccolti mediante la compilazione da parte del personale dirigente e insegnante di schede appositamente elaborate, come tutti gli altri strumenti, dai gruppo di ricerca.

\section{Presentazione dei risultati emersi dalla ricerca svolta nell'area del Litorale sloveno}

Non è possibile riassumere in poche righe $\mathrm{i}$ risultati di questa indagine. I dati si possono analizzare in tanti modi e sono soggetti a diverse interpretazioni. Una lettura completa e realistica potrà essere fatta solamente avendo a disposizione tutte le informazioni metodologiche della ricerca e tutte le informazioni relative alle diverse situazioni socio-linguistiche e ai sistemi scolastici delle aree che hanno partecipato all'indagine.

Una parte dei risultati, senza commento, sono presenti sul sito del progetto (www.progettoinfo.it) mentre i risultati completi e l'analisi critica è stata pubblicata in un volume a conclusione del progetto.

In questa parte dell'intervento presentiamo una breve sintesi di alcuni dati comparativi emersi della ricerca che possono risultare interessanti.

\section{La competenza linguistica nella lingua materna}

I dirigenti erano invitati a valutare le competenze nella lingua materna e nelle lingue europee dei propri insegnanti mentre i docenti si auto-valutavano singolarmente. I dirigenti della scuola dell'isola di San Pietro in Sardegna, dove è parlato il tabarchino, ed i dirigenti delle scuole gallesi (modello ad immersione linguistica) sostengono che il $100 \%$ dei propri insegnanti possiede una competenza linguistica pari alla madrelingua.

I dirigenti delle scuole ladine di Gardena, Badia e Fassa sostengono che il 73\% parla perfettamente la lingua minoritaria e posiziona il rimanente nelle fasce leggermente più basse (livello 6 e 5).

I livelli più bassi di competenza si registrano nelle scuole delle isole germanofone del Friuli dove solo il $10 \%$ dei docenti possiede, secondo i dirigenti, una competenza da madrelingua (ma il restante $90 \%$ viene posizionato nella fascia subito sotto la competenza massima) e le scuole primarie di Alghero in Sardegna, dove solo 13\% risulta essere posizionato nella fascia maggiore e un $63 \%$ è posto nella fascia mediobassa (livello 4). 
Non è possibile confrontare questi dati con i dati emersi dall'autovalutazione degli insegnanti, in quanto la campionatura degli insegnanti rappresenta solamente docenti che si occupano e/o insegnano in lingua minoritaria e che per poter svolgere la propria attività devono obbligatoriamente conoscere perfettamente la lingua minoritaria (mentre i dirigenti hanno valutato tutti i docenti in servizio presso la loro scuola).

La maggioranza dei docenti che insegnano o progettano o svolgono attività in lingua minoritaria si valutano in modo da posizionarsi nella fascia medio-alta, da 5 a 7 (media 5,7) con una punta massima di 7 per i docenti delle scuole ad immersione gallese e minima di 4,5 per le scuole dove il gallese è insegnato come seconda lingua.

I docenti sloveni si posizionano al 6,5 così come i ladini, $i$ catalani, i docenti delle aree germanofone del Friuli ed i catalani di Alghero. Ad abbassare la media è sicuramente l'autovalutazione nelle capacità di scrittura. Infatti, se per la lettura e l'ascolto la valutazione risulta migliore (medio rispettivamente di 5,9 e 6,2) la gran parte dei docenti non si ritiene così abile nella scrittura, in particolare i docenti friulani e i sardi.

\section{La conoscenza e la sperimentazione di nuovi approcci didattici}

Fra i modelli di insegnamento/apprendimento linguistico proposti dal questionario (una persona-una lingua/una lingua - un contesto/immersione linguistica) il più conosciuto risulta essere il modello ad immersione linguistica (conosciuto da 199 docenti su 281). Molti docenti conoscono anche altri modelli e alcuni di questi sono pure menzionati dagli stessi docenti (CLIL, didattica della linguistica integrata, un tempo-una lingua, e altri). Il più utilizzato in genere risulta essere il modello una lingua-un contesto (119 docenti su 281).

Bisogna qui ricordare che le aree oggetto di studio presentano modelli scolastici fra loro molto diversi.

Alcuni di loro prevedono il modello ad immersione linguistica parziale o totale, altri l'insegnamento della lingua e della cultura minoritaria come materia curricolare, altri ancora prevedono l'inserimento della lingua minoritaria insegnata attraverso attività o progetti ad hoc curriculari o extracurriculari.

Alla domanda: "Ti piacerebbe sperimentare nuovi approcci didattici?" sia i dirigenti che i docenti hanno risposto affermativamente. Il $66 \%$ dei docenti ha risposto sì, mentre il rimanente $34 \%$ non è interessato a sperimentare nuove vie per l'apprendimento/insegnamento delle lingue e della lingua materna.

\section{Gli strumenti didattici}

Alla domanda "utilizzi strumenti didattici in lingua minoritaria?" i docenti avevano la possibilità di rispondere con la gradazione mai/a volte/spesso/sempre. Il libro di testo risulta essere lo strumento più utilizzato per la realizzazione dell'insegnamento della lingua minoritaria. Infatti, 58 insegnanti rispondono che lo usano "sempre" e 82 dicono che lo usano "spesso". Articoli su riviste, internet, materiali audiovisivi e multimediali vengono oltremodo utilizzati ma non con la stessa frequenza. 
Gli insegnanti in Slovenia sono in linea con i risultati generali: il 69\% di loro utilizzano "spesso" il libro di testo, il $71 \%$ adotta "a volte" articoli su riviste e/o quotidiani, il 69\% non prende "mai" materiali didattici da internet, a volte (79\%) utilizza materiali audiovisivi e multimediali $(65 \%)$.

Un alto numero di docenti dell'area slovena intervistati sostiene di elaborare "spesso" da sé gli strumenti didattici. La percentuale più alta - 100\% - va ai docenti di lingua catalana ad Alghero, seguiti dagli insegnanti delle scuole ad immersione gallese (77\%).

A volte i docenti della Slovenia utilizzano materiale preparato da altri docenti (risultato in media). Insieme a altri docenti viene preparato "a volte" dal 66\% dei docenti intervistati (anche qui la media generale e più bassa, 56\%), "spesso" $(50 \%)$ viene reperito in biblioteca, a volte $(42 \%)$ dagli istituti di ricerca. In generale il $57,5 \%$ e il $44,7 \%$ dei docenti sostiene di non reperire "mai" materiale didattico da istituti di ricerca, università/associazioni.

\section{Contributo per la promozione della lingua minoritaria}

La domanda che chiedeva di esprimersi sulla valutazione del contributo proprio o della scuola o dell'associazione/ente di appartenenza alla promozione della lingua minoritaria era posta a tutti i soggetti intervistati.

Il 43,9\% dei dirigenti sostiene che la propria scuola svolge una ruolo "totalmente" determinante per la promozione della lingua minoritaria ma solo il $17,9 \%$ dei portatori di interesse sono della stessa opinione, così come il $27 \%$ dei docenti. Specificamente per 1 'area slovena i docenti ritengono la propria attività assolutamente determinante per la promozione della lingua materna, mentre il 50\% dei dirigenti ritiene che l'attività della propria scuola/istituto lo sia completamente.

\section{Comunicazione}

Anche sull'efficacia dei rapporti fra la scuola e le istituzioni che si occupano di lingua minoritaria (istituti di ricerca, musei, associazioni culturali, istituti culturali, ecc.) i pareri non sono concordi. A detta dei dirigenti scolastici sembra che il rapporto fra scuola ed enti sia mediamente efficace $(5,2$ su una scala da 1 a 7$)$ con picchi di 7 per la scuola primaria dell'isola di San Pietro e per le scuole di Fassa.

Le scuole ladine di Gardena e Badia si posizionano sul 6,5 insieme alle scuole slovene e gallesi ad immersione. Il picco più basso lo registrano i dirigenti delle scuole friulane con addirittura un grado di inefficacia di comunicazione pari al 2,5 e le scuole slovene della Carinzia con un 3,2. Interessante notare che solo il $17 \%$ dei portatori di interesse si posiziona sul livello massimo di efficacia, il $21,4 \%$ si posiziona parimenti sul livello 5 e 6 . Una percentuale leggermente più alta dei docenti $(22,2 \%)$ ritiene la comunicazione totalmente efficace. Il $23 \%$ si posiziona al livello 6 e il 14,4 al livello 5. 


\section{Le motivazione di fondo}

Le motivazioni che spingono i docenti a promuovere la lingua minoritaria sono "spesso" di tipo culturale e di carattere personale, rispettivamente per il $66,8 \%$ e per $61,2 \%$ dei docenti.

La motivazione politica è la meno gettonata con il $12,1 \%$ dei docenti che hanno risposto "spesso" e il 12,8\% "abbastanza". Anche le motivazioni economiche non sono le ragioni dell'impegno per l'insegnamento della lingua minoritaria (la maggioranza $34,2 \%$ - ritiene queste "poco" motivanti).

\section{La sicurezza nella programmazione}

Interessanti le risposte relative alle domande sulla sicurezza nella programmazione e sul grado di soddisfazione della propria attività didattica.

La maggioranza dei docenti ladini si posiziona nelle fasce medio-alte (livelli 4, 5 e 6) mentre solo il $13 \%$ si sente completamente sicuro, a differenza del $50 \%$ degli insegnanti di lingua friulana e dell' $85 \%$ degli insegnanti della Slovenia e gallesi nelle scuole ad immersione. Il $49 \%$ dei docenti si ritiene soddisfatto (livello 6) ma completamente soddisfatto solo l'8\% degli intervistati (media 5,31). I più soddisfatti sono ancora gli insegnanti di gallese nelle scuole ad immersione dove $46 \%$ si ritiene completamente soddisfatto. I meno soddisfatti sono gli insegnanti di friulano e di catalano (rispettivamente con la media 4,60 e di 4,71).

\section{I risultati dell'attività didattica}

Ai portatori di interesse veniva chiesto come percepiscono i risultati dell'attività didattica in lingua minoritaria attuata nella scuola primaria del proprio territorio. Il $37 \%$ degli intervistati pone un livello 5 in una scala da un minimo di 1 che equivale ad un risultato negativo ad un massimo di 7 per la completa positività dei risultati. Solo l'11\% dei portatori di interesse è completamente soddisfatto delle attività della propria scuola/istituto.

\section{Attuazione della normativa}

Interessante intersecare i risultati dei dati dei portatori di interesse e dei dirigenti alla domanda: "In che misura vengono realizzate nella scuola primaria del suo territorio le possibilità di promozione della lingua minoritaria previste dalla legge?". La percentuale più alta $(24,1 \%)$ dei portatori di interesse dà una valutazione al livello $4, \mathrm{i}$ rimanenti si posizionano equamente sulla scala di valutazione al livello 2 e 3 e 5 e 6 $(13,8 \%)$. La maggioranza dei dirigenti scolastici si pone invece sui livelli 5, 6 e 7, ad eccezione dei dirigenti friulani e sardi che ritengono che non siano attuate tutte le misure previste dalla normativa.

Pur verificando la diversità del grado di presenza della lingua minoritaria nei diversi sistemi scolastici si sono potuti comunque trovare degli elementi comuni come, ad esempio, puntare sulle competenze didattiche e linguistiche degli insegnanti tramite 
percorsi formativi professionalizzanti volti a migliorare la qualità dell'insegnamento e a sperimentare nuovi approcci metodologici che coinvolgano l'intera gamma delle discipline in modo da proporre interventi incisivi a livello pluridisciplinare e plurilinguistico; offrire una maggiore e diversificata gamma di strumenti didattici in lingua, la cui carenza quantitativa è testimoniata da tutte le realtà. Favorire la presa di coscienza negli alunni della propria identità linguistica e culturale attraverso la valutazione della lingua minoritaria, l'integrazione e l'accettazione della diversità, il confronto e l'educazione alla convivenza. Ed inoltre fattore emerso come esigenza per tutte le realtà è quello di dare maggiore visibilità a quanto viene fatto e prodotto, coinvolgendo il territorio e le istituzioni nel progetto di valorizzazione della lingua minoritaria

\section{Il corso di formazione - Un modello di formazione europeo}

Dall'analisi dei dati emersi dalla ricerca il gruppo di ricercatori del progetto INFO ha elaborato un modello sperimentale di formazione per insegnanti delle scuole primarie che insegnano e/o utilizzano la lingua minoritaria nella propria attività didattica.

La scelta metodologica del gruppo di progetto è caduta su un corso in presenza residenziale. Tale scelta è stata operata per poter dare particolare risalto ad alcuni elementi di qualità ritenuti fondamentali in questo contesto formativo. Come ad esempio, la provenienza dei corsisti da più aree di lingua minoritaria che permette la creazione di un contesto di condivisione e formazione in dimensione europea.

Il corso di formazione per inseganti è stato strutturato secondo un percorso che ha visto una prima settimana di formazione tenutasi a Bolzano presso la sede dell'Istituto Pedagogico Ladino, a cui è seguito il lavoro sviluppato direttamente dagli insegnanti (corsisti) con i loro alunni. Il lavoro è stato supportato da periodici contatti con i tutor dei partner del progetto. I risultati dei lavori sviluppati sono poi stati esposti e discussi in un ulteriore incontro di Follow-up ad Ortisei in Val Gardena.

I contenuti del corso sono stati discussi e concordati nelle riunioni di progetto, durante le quali è stata fatta una scelta dei docenti da coinvolgere, sulla base degli ambiti di specializzazione e delle esperienze pregresse dei docenti. Al corso hanno partecipato 21 insegnanti, in media tre per ogni realtà minoritaria rappresentata nel progetto. La settimana iniziale di formazione ha visto la trattazione degli argomenti relativi alla politica linguistica, allo studio degli aspetti legali relativi alla tutela in Europa della minoranza linguistica, al project management in teaching, all'educazione plurilingue e all'insegnamento della lingua minoritaria con momenti di laboratorio per la produzione di strumenti e materiali didattici.

Tutto il percorso è stato monitorato con strumenti di valutazione. Nel corso nel follow-up gli insegnanti hanno potuto confrontarsi sul lavoro svolto da ognuno con i loro alunni, infatti sono stati presentati e discussi i progetti sviluppati nelle scuole su sollecitazione dal corso di formazione. Anche questo momento ha visto il monitoraggio con strumenti di valutazione con cui il gruppo di progetto ha voluto constatare la messa in opera dei saperi trasmessi durante il corso. Dalla lettura della valutazione del corso e del follow-up è stato possibile osservare che per molti corsisti il percorso è stato decisamente innovativo, e in particolare, motivo di sprono per attuare attività di 
promozione della lingua minoritaria in un'ottica di didattica del plurilinguismo. In particolare, là dove la promozione della lingua minoritaria è demandata alla volontà dei singoli o ai propri interessi personali.

Pur essendo consapevoli della diversità si possono individuare alcuni punti chiave comuni ai vari progetti che possono essere ripresi e adattati ad altri contesti linguistici minoritari. Fra questi si possono indicare le scelte di un approccio metodologico di tipo comunicativo che pone l'accento sulle abilità orali e sul saper fare e si propone di migliorare lo standard della lingua parlata; l'uso di modalità e tecniche d'insegnamento innovative improntate alla flessibilità, attenta agli stili individuali e alle strategie di apprendimento degli alunni; la didattica laboratoriale come modalità di lavoro adattato alla scuola primaria; il ricorso ad attività motivanti didatticamente e affettivamente per coinvolgere il bambino in prima persona, sollecitare il suo interesse per la propria lingua e la comunità verso altre lingue e culture; l'uso di una grande varietà di materiali, la maggior parte dei quali prodotto autonomamente dai corsisti per rispondere in modo mirato alle diverse esigenze dei bambini e per sollecitare la loro curiosità verso le proprie origini e verso le proprie tradizioni culturali; l'utilizzo delle nuove tecnologie in tutti i progetti a sostegno di una maggiore interazione tra $\mathrm{i}$ bambini; la visione della lingua come veicolo privilegiato di cultura, come stimolo per il plurilinguismo, apprendimento linguistico, ma anche educazione interculturale ed in fine, la consapevolezza che l'integrazione linguistica e l'integrazione con gli altri linguaggi sono alla base di un percorso che dia dignità e forza alla lingua minoritaria verso un curriculo veramente plurilingue.

\section{Conclusioni}

Il corso di formazione proposto ai docenti nel quadro del progetto INFO, si propone come modello estensibile ed adattabile alle singole realtà minoritarie regionali in Europa.

Per la sua natura di prototipo e di modello, tale corso si prospetta di carattere generale e comprensivo di diversi aspetti, frutto di una puntuale ed estesa rilevazione dei bisogni sul campo.

Le competenze generali e specifiche da perseguire tramite il corso e l'azione progettuale connessa, afferivano all'area del sapere, del saper fare e del saper essere ed andavano ad interessare la natura stessa dell'azione educativa in ambito minoritario e plurilingue.

Per la sua ricchezza di contenuti e per la qualità dei referenti, il corso può essere considerato una solida base di conoscenza ed un potente stimolo alla ricerca azione nella realtà scolastica dei partecipanti stessi.

$\mathrm{Si}$ auspica, come ricaduta del progetto, oltre alla disseminazione delle buone pratiche e del modello di formazione, una diffusione dell'esperienza attuata, al fine di perseguire un maggior impegno dei sistemi politico-amministrativi delle varie realtà scolastiche europee per la promozione delle lingue minoritarie in contesti plurilingui, affinché il principio dell' "unità nella diversità" si realizzi anche in quelle situazioni 
sociolinguistiche ove le lingue regionali cercano di imporsi a fronte della globalizzazione avanzante.

\section{Bibliografia}

Balboni, P.E. (1999). Educazione Bilingue. Perugia: Guerra.

Balboni, P. E, Coonan, C. M., Garotti Ricci, F. (2001). Lingue straniere nella scuola dell'infanzia. Perugia: Guerra.

Blassnig, E., Mayring, Ph., Vovk Korže, A., Korže, D., Bernjak, E., Pišot, R., Zudič Antonič, N., Zorman, A., Hieden, J. (a cura di.) Abl, K. H. (ur.) (2005). Input-Evaluation des Projekts Förderung von Minderheitensprachen im mehrsprachigen Raum in der Lehrerbildung: Endbericht, (Förderung von Minderheitensprachen, Bd. 2). Klagenfurt: Pädagogische Akademie des Bundes in Kärnten.

Čok, L. (2001). The role of the minority languages in Slovene education policy: the case of Slovene Istria. Geogr. Slov., 2001, anno 34, n. 1, 253-266.

Čok, L. (2003). Cultures and languages of the environment : the role of minority language in slovene education policy. V: Schiavi Fachin, Silvana (a cura di). L'educazione plurilingue: dalla ricercha di base alla pratica didattica. Udine: Forum, 2003, 59-69.

Zudič Antonič, Nives, Zorman, Anja (2006). Predstavitev pogledov otrok in staršev iz različnih držav na nekatere vidike učenja in poučevanja manjšinskih jezikov. Šol. Polje anno 17, n. 1/2, 127-142.

Zudič Antonič, Nives, Malčič, Metka (2007). The schools of the Italian minority on the Slovene coast: Slovenia. In: Rasom, Olimpia (a cura di). Info project: designing a training model for teachers working in multilingual areas with minority language. Part 1, The research and the European training model. Istitut Pedagogich Ladin, 39-42.

Zudič Antonič, Nives, Malčič, Metka (2007). The needs. In: Rasom, Olimpia (a cura di). Info project: designing a training model for teachers working in multilingual areas with minority language. Part 1, The research and the European training model. Istitut Pedagogich Ladin, 84-87. 
Metodički obzori 6(2011)2

Izvorni znanstveni rad

UDK: 371.13:[378.7:811.131.1]

Primljeno: 23. 11. 2010.

\title{
PREDSTAVLJANJE MODELA IZOBRAZBE ZA NASTAVNIKE KOJI RADE U VIŠEJEZIČNOM PODRUČJU UZ PRISUTNOST MANJINSKOG JEZIKA
}

\author{
Doc. dr. Nives Zudič Antonič, \\ Univerza na Primorskem, \\ Fakulteta za humanistične študije, Koper (Slovenija) \\ e-mail: nives.zudic.antonic@ @hs.upr.si
}

\section{Sa žetak}

Ovim radom prikazuju se rezultati međunarodnog istraživanja realiziranog $u$ području projekta Comenius 2.1. INFO - Identifikacija modela izobrazbe za nastavnike koji rade u višejezičnom području uz prisutnost manjinskog jezika (2005-07).

Svrha projekta bila je identificirati model obrazovanja koji se temelji na pokazateljima kvalitete, a koji je povezan $\mathrm{s}$ novim stručnim kompetencijama, prikladnim posebnostima višejezičnih područja, odgovarajućim nastavnim oblicima rada i metodikom višejezičnosti. U području projekta bile su istaknute obrazovne potrebe u sociokulturnom kontekstu, potrebe sustava i osobne potrebe nastavnika. Analizom tih potreba identificirao se referentni okvir kompetencija, sposobnosti i ponašanja koje su pokazali nastavnici koji rade u višejezičnoj sredini.

Projekt je nastao kako bi se izašlo u susret stvarnim i zajedničkim potrebama nastavnika, pripadnika jezičnih povijesnih europskih manjina. Bilingvizam, ili, ponekad, višejezičnost, koji karakterizira govornike jezične manjine i koji utječe na jezične kompetencije tih istih govornika, obuhvaća posebna znanja i pristupe nastavnika koji rade u školskim institucijama.

Posljednjih je godina osnažila zaštita manjina i to zahvaljujući njihovom samounapređenju te uzlaznoj osjetljivosti u odnosu na manje rasprostranjene jezike koji predstavljaju bogatstvo u europskom kulturnom pregledu.

No postoje mnoge različitosti u kontekstu normativnih europskih sustava koji, na izrazito raznolik način, uključuju poučavanje materinjeg jezika unutar školskih institucija.

Neke europske zemlje osiguravaju poučavanje materinjeg jezika od osnovne škole, drugi ga promatraju kao izvankurikulumsku mogućnost ili planiranje, a treći ga isključuju iz cjelokupne školske prakse.

Sve do sada u jednojezičnoj prirodi gdje se poučavaju svi nastavni predmeti, većinom u nacionalnom jeziku, a radi uvođenja manjinskog jezika u školski sustav, nastavniku su bile potrebne posebne kompetencije i znanja što mu je omogućavalo promicanje materinjeg jezik u višejezičnom kurikularnom kontekstu. Ukoliko pismenost u materinjem jeziku za manjinu ne predstavlja realno dostižan cilj u višejezičnom kontekstu poput suvremenog, školski sustavi u području gdje postoji manje rasprostranjen jezik moraju znati pronaći i provoditi metodiku i metodologiju koja, prije svega, dopušta promicanje materinjeg jezika kao i druge jezike prisutne na određenom području, u društvenom i kulturnom kontekstu, kao što je slučaj s ostalim europskim jezicima.

Cilj projekta je identificirati i realizirati model stručne izobrazbe nastavnika, koji, naglašavajući elemente stručne kvalitete, omogućava nastavnicima uporabu 
metodičkih i metodologijskih instrumenata, znanja i stavova i uporabu pristupa i strategija kojima će na najbolji način realizirati didaktičku praksu i u potpunosti promicati mogućnost razvoja učenika kao govornika onih jezika koji su manje rasprostranjeni.

Projekt se razvija u suradnji nekoliko europskih partnera iz područja gdje postoje jezične manjine. $\mathrm{S}$ obzirom na dobivene rezultate $\mathrm{u}$ istraživanju, obrađene $\mathrm{u}$ sklopu projekta, bilo je moguće izraditi model izobrazbe. Ovaj model nastoji predložiti institucijama koje obrazuju i vrše izobrazbu za nastavno osoblje Smjernice kako bi se strukturirao poseban tečaj za nastavnike osnovnih škola koji rade u višejezičnom području uz prisutnost manjinskog jezika. Smjernicama se sadržaje tečaja orijentira prema realnim potrebama nastavnika koji žele i trebaju promicati i realizirati projekt, aktivnost ili tečaj u poučavanju manjinskog jezika, za one koji će se susresti $\mathrm{s}$ višejezičnim metodičkim pristupom uzimajući u obzir materinji jezik učenika (u slučaju da se radi o manjinskom jeziku) ili samo za one koji se žele profesionalno uskladiti s europskim nastavnim standardima koji se sve više kreću prema metodici višejezičnosti.

Ključne riječi: jezik manjine, talijanski, Comenius 2.1, predavači, obrazovanje učitelja, višejezičnost, tečaj edukacije 